01

\title{
Реконструкция архитектуры связей в цепочке из трех однонаправленно связанных систем методом причинности по Грейнджеру
}

\author{
(C) М.В. Корнилов ${ }^{1,2}$, И.В. Сысоев ${ }^{1,2}$ \\ ${ }^{1}$ Саратовский фрилиал Института радиотехники и электроники \\ им. В.А. Котельникова РАН, Саратов, Россия \\ ${ }^{2}$ Саратовский национальный исследовательский государственный \\ университет им. Н.Г. Чернышевского, Саратов, Россия \\ E-mail: kornilovmv@gmail.com
}

Поступило в Редакцию 9 января 2018 г.

Предложена методика реконструкции связей в цепочке однонаправленно связанных осцилляторов на основе проведения трех тестов, оценивающих прямое и опосредованное взаимодействие методом условной причинности по Грейнджеру, с использованием прогностических моделей с полиномиальной нелинейностью. Показано, что данный подход позволяет более чем в $80 \%$ случаев верно определять архитектуру связей.

DOI: 10.21883/PJTF.2018.10.46103.17201

В настоящее время существуют различные подходы к детектированию направленной связанности между элементами сложных систем по их экспериментальным сигналам. Один из самых популярных метод причинности по Грейнджеру [1]. Если подсистем больше двух, дополнительно к проблеме детектирования наличия и направления связей возникает проблема различения прямого и опосредованного взаимодействия. В частности, она представляет большой практический интерес для задач радиотехники, нейрофизиологии и климатологии. Общая идея решения данной проблемы известна: следует использовать прогностические модели, при построении которых учитывается информация не только от двух подсистем (ведомой и ведущей), но от нескольких (такой подход в [2] получил название условной причинности по Грейнджеру). Однако ее практическое воплощение, особенно для 
нелинейных систем, оказывается затруднительным по многим причинам, главная из которых - недостаток объема имеющихся экспериментальных данных для надежной оценки всех коэффициентов [3]. Недостаточно высокая частота выборки, малая общая длина записи и, как следствие, малое доступное число колебаний и недоступность для измерения части подсистем не дают возможности решить проблему в общем, однако отдельные успешные частные решения возможны [4].

В настоящей работе рассматривается задача о реконструкции архитектуры связей в цепочке из трех однонаправленно связанных подсистем, демонстрирующих колебания с одним или несколькими основными временны́ми масштабами, методом нелинейной причинности по Грейнджеру с использованием моделей в виде отображений последования с полиномиальными нелинейностями в соответствии с [2]. Цель работы - определить, насколько метод способен верно распознать архитектуру связей и, что наиболее существенно, различить прямое и опосредованное взаимодействие при разных уровнях связи (от очень слабых и не приводящих к существенным изменениям в динамике ведомых систем до достаточно сильных, ведущих к синхронизации).

Пусть в нашем распоряжении имеются скалярные временны́е ряды $\left\{x_{n}\right\}_{n-1}^{N},\left\{y_{n}\right\}_{n=1}^{N}$ и $\left\{z_{n}\right\}_{n-1}^{N}$ от трех систем $X, Y, Z$ длиной $N$ отсчетов каждый. При этом система $X$ считается ведомой и предполагается, что она не может воздействовать ни на $Y$, ни на $Z$. Система $Y$ (промежуточная система) может воздействовать только на $X$, а система $Z$ может воздействовать на $X$ как непосредственно, так и опосредованно через $Y$ или не воздействовать вообще (используя термин „цепочка“, мы в первую очередь подразумеваем, что не может быть обратных связей вида $X \rightarrow Y$ или $Y \rightarrow Z$ или $X \rightarrow Z$, т.е. если воздействие и распространяется, то в одну строну). Всего, таким образом, возможно восемь случаев взаимодействия:

1) $Z \rightarrow Y \rightarrow X$ без непосредственного воздействия $Z$ на $X$ (опосредованная связь);

2) $Z \rightarrow X \wedge Z \rightarrow Y \rightarrow X-$ случай прямой и опосредованной связи одновременно;

3) $Z \rightarrow Y \wedge Z \rightarrow X$, но без воздействия $Y$ на $X$, т.е. только прямая связь;

4) $Z \rightarrow X \wedge Y \rightarrow X-$ при отсутствии опосредованного взаимодействия;

5) отсутствие связи между $Z$ и $X$, но наличие связи $Y \rightarrow X$;

Письма в ЖТФ, 2018, том 44, вып. 10 
6) $Z \rightarrow X-$ прямая связь, без искажения сигнала системы $Y$ сигналом системы $Z$;

7) отсутствие связи между $Z$ и $X$, но наличие связи $Z \rightarrow Y$ (система $X$ изолирована);

8) отсутствие каких-либо связей, все системы изолированы.

Такая постановка задачи может соответствовать системе беспроводных сенсоров, передающих информацию о напряжении свода здания или уровне задымления по цепочке [5], или соображениям анатомии, когда существуют восходящие связи от органов чувств к глубоким структурам мозга и далее в кору.

Поскольку тест на причинность по Грейнджеру может ответить только на вопрос о том, есть ли значимая связь между системами в заранее заданном направлении или нет, чтобы определить, какой из восьми рассмотренных случаев имеет место, нужно провести как минимум $\log _{2} 8=3$ тестирования на связанность. Для исследования связи $Z \rightarrow Y, Z \rightarrow X$ и $Y \rightarrow X$ наиболее очевидный подход состоит в построении прогностических моделей вида

$$
\begin{gathered}
x_{n+\tau}^{\prime}=f_{x}\left(\mathbf{x}_{n}\right), x_{n+\tau \mid Y}^{\prime}=f_{x y}\left(\mathbf{x}_{n}, \mathbf{y}_{n}\right), x_{n+\tau \mid Z}^{\prime}=f_{x z}\left(\mathbf{x}_{n}, \mathbf{z}_{n}\right), \\
y_{n+\tau}^{\prime}=f_{y}\left(\mathbf{y}_{n}\right), y_{n+\tau \mid Z}^{\prime}=f_{y z}\left(\mathbf{y}_{n}, \mathbf{z}_{n}\right)
\end{gathered}
$$

и дальнейшем расчете улучшения прогноза по формулам

$$
P I_{y \rightarrow x}=1-\frac{\varepsilon_{x y}^{2}}{\varepsilon_{x}^{2}}, P I_{z \rightarrow x}=1-\frac{\varepsilon_{x z}^{2}}{\varepsilon_{x}^{2}}, P I_{z \rightarrow y}=1-\frac{\varepsilon_{y z}^{2}}{\varepsilon_{y}^{2}} .
$$

Здесь $\mathbf{x}_{n}, \mathbf{y}_{n}$ и $\mathbf{z}_{n}$ - реконструированные методом временнб́х задержек [6] по скалярным временны́м рядам векторы состояния, функции $f_{x}, f_{x y}, f_{x z}, f_{y}$ и $f_{y z}$ - полиномы общего вида, как предложено в [2], $\tau$ - дальность прогноза, $x_{n}^{\prime}$ и др. - предсказанные значения, соответствующие измеренным значениям $x_{n}, P I-$ показатели улучшения прогноза, $\varepsilon$ - среднеквадратичные ошибки аппроксимации.

Однако такой подход не может в действительности решить поставленную задачу, потому что при наличии опосредованного воздействия $Z \rightarrow Y \rightarrow X$ с его помощью будет обнаружена также связь $Z \rightarrow X$, так как $X$ содержит информацию о динамике $Z$. А при воздействии $Z \rightarrow Y$ и $Z \rightarrow X$ может быть обнаружено воздействие $Y \rightarrow X$, поскольку $Y$ может содержать часть информации от $Z$, что поможет предсказать $X$. Чтобы

Письма в ЖТФ, 2018, том 44, вып. 10 
Соответствие искомой архитектуры связей и выводов о наличии связи при идеальной чувствительности и специфичности предложенной методики (3), (4)

\begin{tabular}{|c|c|c|c|c|}
\hline $\begin{array}{c}\text { № } \\
n / n\end{array}$ & $\begin{array}{c}\text { Вид } \\
\text { взаимодействия }\end{array}$ & $P I_{z \rightarrow y}$ & $P I_{y \rightarrow x \mid z}$ & $P I_{z \rightarrow x \mid y}$ \\
\hline 1 & $Z \rightarrow Y \rightarrow X$ & 1 & 1 & 0 \\
\hline 2 & $Z \rightarrow X \wedge Z \rightarrow Y \rightarrow X$ & 1 & 1 & 1 \\
\hline 3 & $Z \rightarrow Y \wedge Z \rightarrow X$ & 1 & 0 & 1 \\
\hline 4 & $Z \rightarrow X \wedge Y \rightarrow X$ & 0 & 1 & 1 \\
\hline 5 & $Z \wedge Y \rightarrow X$ & 0 & 1 & 0 \\
\hline 6 & $Z \rightarrow X \wedge Y$ & 0 & 0 & 1 \\
\hline 7 & $Z \rightarrow Y \wedge X$ & 1 & 0 & 0 \\
\hline 8 & $Z \wedge Y \wedge X$ & 0 & 0 & 0 \\
\hline
\end{tabular}

Примечание. Белый фон (отвечает $P I=1)$ соответствует значимым выводам о наличии связи, серый (отвечает $P I=0)-$ об отсутствии.

решить проблему, нужно вместо простой причинности по Грейнджеру, когда системы рассматриваются попарно, перейти к условной [2], построив модели вида

$$
\begin{gathered}
x_{n+\tau \mid Y}^{\prime}=f_{x y}\left(\mathbf{x}_{n}, \mathbf{y}_{n}\right), x_{n+\tau \mid Z}^{\prime}=f_{x z}\left(\mathbf{x}_{n}, \mathbf{z}_{n}\right), x_{n+\tau \mid Y, Z}^{\prime}=f_{x y z}\left(\mathbf{x}_{n}, \mathbf{y}_{n}, \mathbf{z}_{n}\right) \\
y_{n+\tau}^{\prime}=f_{y}\left(\mathbf{y}_{n}\right), \quad y_{n+\tau \mid Z}^{\prime}=\left(\mathbf{y}_{n}, \mathbf{z}_{n}\right)
\end{gathered}
$$

и рассчитав улучшение прогноза по формулам

$$
P I_{y \rightarrow x \mid z}=1-\frac{\varepsilon_{x y z}^{2}}{\varepsilon_{x z}^{2}}, P I_{z \rightarrow x \mid y}=1-\frac{\varepsilon_{x y z}^{2}}{\varepsilon_{x y}^{2}}, P I_{z \rightarrow y}=1-\frac{\varepsilon_{y z}^{2}}{\varepsilon_{y}^{2}} .
$$

Результаты, которые должны получиться с использованием такой методики в „идеальной“ ситуации, показаны в таблице. Видно, что для каждого из восьми выбранных случаев возможного взаимодействия существует только одна комбинация результатов методики, т. е. достигается взаимно однозначное соответствие.

В реальной ситуации достижение $P I=0$ (при отсутствии связей) и $P I=1$ (при наличии) или даже близких к ним с точностью вычислений значений невозможно как по причине конечности частоты и объема выборки [7], так и из-за несовершенства моделей [8]. Поэтому принято

Письма в ЖТФ, 2018, том 44, вып. 10 
считать, что связь обнаружена, если полученное значение выше тестового уровня, для расчета которого в настоящей работе использовался широко распространенный подход, основанный на генерации суррогатных временны́х рядов [9]. Генерировалось 100 реализаций суррогатных рядов тестируемых систем с теми же параметрами при различных начальных условиях, но при отсутствии связи, по ним оценивался 95\% уровень значимости. В [10] было показано, что такой способ дает удовлетворительные результаты и примерно эквивалентен некоторым другим распространенным подходам.

Размерность вектора состояния и степень полинома в моделях (3) выбирались по критерию Шварца [11]. Параметры (лаг вложения $l$ и дальность прогноза $\tau$ ) выбирались с учетом основного временно́го масштаба ведомой системы, как предложено в [12].

Для тестирования в качестве систем $X, Y$ и $Z$ были выбраны широко известные эталонные модели радиотехнических и оптических систем: уравнения генератора с 1.5 степенями свободы [13] и система Икеды [14]. Параметры систем и связи были выбраны и введены в соответствии с уравнениями

$$
\begin{aligned}
& \left\{\begin{array}{l}
\dot{x}_{1}=\left(F\left(x_{3}, 26\right)-x_{1}\right) / 3+k_{y x}\left(y_{1}-x_{1}\right)+k_{z x}\left(z_{1}-x_{1}\right), \\
\dot{x}_{2}=x_{1}-x_{3}, \\
\dot{x}_{3}=x_{2}-0.21 x_{3},
\end{array}\right. \\
& \left\{\begin{array}{l}
\dot{y}_{1}\left(F\left(y_{3}, 28\right)-y_{1}\right) / 3+k_{z y}\left(z_{1}-y_{1}\right), \\
\dot{y}_{2}=y_{1}-y_{3}, \\
\dot{y}_{3}=y_{2}-0.22 y_{3},
\end{array}\right. \\
& \left\{\begin{array}{l}
\dot{z}_{1}=\left(F\left(z_{3}, 30\right)-z_{1}\right) / 3, \\
\dot{z}_{2}=z_{1}-z_{3}, \\
\dot{z}_{3}=z_{2}-0.23 z_{3},
\end{array} \quad F(x, M)=M x \exp \left(x^{2}\right)\right.
\end{aligned}
$$

И

$$
\begin{gathered}
\dot{x}(t)=-x(t)+15 \sin \left(x(t-3)-x_{0}\right)+k_{y x}(y(t)-x(t))+k_{z x}(z(t)-x(t)), \\
\dot{y}(t)=-y(t)+20 \sin \left(y(t-2)-y_{0}\right)+k_{z y}(z(t)-y(t)), \\
\dot{z}(t)=-z(t)+25 \sin \left(z(t-4)-z_{0}\right) .
\end{gathered}
$$



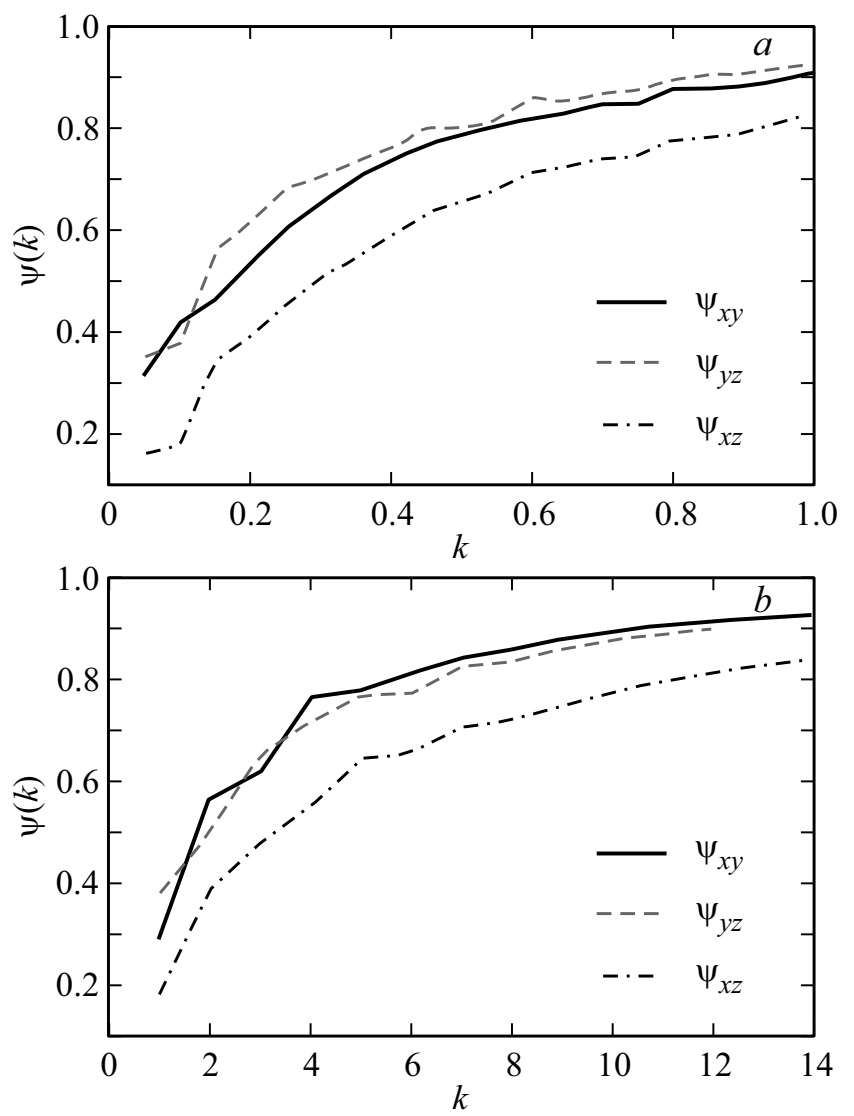

Рис. 1. Зависимости значений коэффициентов фазовой синхронизации $\psi_{x y}, \psi_{x z}$ и $\psi_{y z}$ от коэффициентов связи $k_{y x}=k_{z y}=k: a-$ между автогенераторами с 1.5 степенями свободы в уравнении (5), $b$ - между системами Икеды (6). Коэффициент $k_{z x}=0$, что соответствовало архитектуре 1 .

Для системы (5) координаты $x_{1}, y_{1}, z_{1}$ считались наблюдаемыми. Все или некоторые коэффициенты связи $k_{y x}, k_{z x}$ и $k_{z y}$ могли обнуляться, чтобы рассмотреть различные архитектуры связи. Если соответствующий коэффициент не обнулялся, он изменялся в широком диапазоне, как показано на рис. $1, a$. Были выбраны три значения $k: 0.15,0.4$ и 0.7 ,

Письма в ЖТФ, 2018, том 44, вып. 10 
для которых проводилось исследование с усреднением результата по 100 реализациям, полученным при различных начальных условиях.

Результаты для связанных автогенераторов представлены на рис. 2, $a$, где показано, что в среднем наилучшую работоспособность метод демонстрирует в случае, когда $k=0.4$. Однако при наличии прямого и опосредованного взаимодействия $(Z \rightarrow X \wedge Z \rightarrow Y \rightarrow X)$ наилучшие результаты достигаются при $k=0.7$. Следует отметить, что наиболее сложным оказался тест для оценки $P I_{y \rightarrow x \mid z}$ в случае $Z \rightarrow Y \wedge Z \rightarrow X$, где наихудшие результаты достигаются при $k=0.7$. По остальным возможным взаимодействиям метод демонстрирует ожидаемые результаты.

Аналогичным образом численный эксперимент проводился и для связанных однонаправленной связью систем Икеды (6). На основании зависимости, представленной на рис. $1, b$, были выбраны три значения $k: 2,5,10$, для которых проводилось три теста с усреднением по 100 реализациям. Результаты численного эксперимента представлены на рис. $2, b$. Метод демонстрирует наилучшую работоспособность для $k=2$. При этом ошибочные выводы о наличии направленной связи наблюдаются для оценок $P I_{y \rightarrow x \mid z}$ и $P I_{z \rightarrow x \mid y}$ в случаях $Z \rightarrow Y \wedge Z \rightarrow X$ и $Z \rightarrow Y \rightarrow X$ соответственно. По остальным возможным взаимодействиям метод демонстрирует ожидаемые результаты.

В результате численного эксперимента было показано, что с помощью предложенной методики по скалярным временны́м рядам сложных колебаний трех систем с выраженным характерным временны́м масштабом можно надежно различить прямое и опосредованное воздействие в $82 \%$ случаев, используя модели, построенные с учетом временны́х масштабов сигналов. Эффективность метода выше для некоторых „средних“ коэффициентов связи, когда наличие связей заметно модифицирует динамику ведомой системы, но при этом не приводит к синхронизации. Полученный результат ценен тем, что оказывается возможным различать источник и путь распространения сигнала, не имея априорных знаний о системе, его генерирующей.

Наибольшую сложность представляет случай наличия одновременно прямого и опосредованного взаимодействия. Такой случай часто не может быть отделен от случая наличия только прямого воздействия первой системы в цепочке на две остальные: конечную и промежуточную. Это объясняется слабым вкладом опосредованного воздействия при наличии прямого в динамику третьей системы. Однако на практике ошибки такого рода, как правило, наименее критичны. Также показано,

Письма в ЖТФ, 2018, том 44, вып. 10 


\begin{tabular}{|c|c|c|c|c|c|c|c|c|c|}
\hline \multirow[b]{2}{*}{$Z \rightarrow Y \rightarrow X$} & \multicolumn{3}{|c|}{$k=0.15$} & \multicolumn{3}{|c|}{$k=0.4$} & \multicolumn{3}{|c|}{$k=0.7$} \\
\hline & 0.99 & 1.0 & 1.0 & 1.0 & 1.0 & 0.79 & 1.0 & 1.0 & 0.46 \\
\hline$Z \rightarrow X \wedge Z \rightarrow Y \rightarrow X$ & 0.89 & 0.72 & 0.95 & 1.0 & 0.94 & 1.0 & 1.0 & 1.0 & 1.0 \\
\hline$Z \rightarrow Y \wedge Z \rightarrow X$ & 0.97 & 0.96 & 1.0 & 1.0 & 0.42 & 1.0 & 1.0 & 0.15 & 1.0 \\
\hline$Z \rightarrow X \wedge Y \rightarrow X$ & 0.84 & 0.85 & 0.89 & 0.89 & 1.0 & 1.0 & 0.95 & 1.0 & 1.0 \\
\hline$Z \wedge Y \rightarrow X$ & 1.0 & 1.0 & 0.97 & 1.0 & 1.0 & 1.0 & 0.72 & 1.0 & 0.91 \\
\hline$Z \rightarrow X \wedge Y$ & 0.92 & 1.0 & 1.0 & 0.74 & 1.0 & 1.0 & 0.94 & 0.86 & 1.0 \\
\hline$Z \rightarrow Y \wedge X$ & 1.0 & 0.92 & 1.0 & 1.0 & 1.0 & 1.0 & 1.0 & 0.9 & 0.96 \\
\hline$Z \wedge Y \wedge X$ & 0.99 & 0.95 & 0.96 & 0.96 & 0.98 & 0.98 & 0.95 & 0.89 & 0.97 \\
\hline
\end{tabular}

Рис. 2. Процент верных выводов о наличии (отсутствии) связи между системами по выбранным восьми видам взаимодействия для автогенераторов с 1.5 степенями свободы $(a)$ и для систем Икеды $(b)$. Для каждого типа прогностической модели градациями серого (белый $-100 \%$, черный $-0 \%$ ) и значениями отмечены отношения верных выводов (в соответствии с таблицей) к общему количеству тестов. 
Рис. 2 (продолжение). 
что использованный подход пригоден для выявления факта отсутствия связей в цепочке или ее разрыва, поскольку он позволяет определить отсутствующие связи. При таком использовании его эффективность достигает $90 \%$ и более.

Результаты, полученные при исследовании цепочек из двух различных типов эталонных осцилляторов, в основном сходны, хотя для систем автогенераторов с 1.5 степенями свободы эффективность метода в среднем выше, что может объясняться более простой динамикой отдельных систем (меньшая размерность) и более выраженным временны́м масштабом колебаний. Это позволяет лучше подобрать параметры метода на основании ранее разработанных в рамках проекта критериев.

Работа выполнена при финансовой поддержке Российского научного фонда грант (14-12-00291).

\section{Список литературы}

[1] Granger C.W.J. // Econometrica. 1969. V. 37. N 3. P. 424-438.

[2] Chen Y., Rangarajan G., Feng J., Ding M. // Phys. Lett. A. 2004. V. 324. N 1. P. 26-35.

[3] Smirnov D.A., Bezruchko B.P. // Europhys. Lett. 2012. V. 100. N 1. P. 10005.

[4] Angelini L., Pellicoro M., Stramaglia S. // Phys. Lett. A. 2009. V. 373. N 29. P. 2467-2470.

[5] Yick J., Mukherjee B., Ghosal D. // Comput. Networks. 2008. V. 52. N 12. P. 2292-2330.

[6] Packard N., Crutchfield J., Farmer J., Shaw R. // Phys. Rev. Lett. 1980. V. 45. N 9. P. 712-716.

[7] Крылов С.Н., Смирнов Д.А., Осипов Г.В., Безручко Б.П. // Письма в ЖТФ. 2015. T. 41. B. 11. C. $94-102$.

[8] Smirnov D.A. // Phys. Rev. E. 2014. V. 90. N 6. P. 062921.

[9] Theiler J., Eubank S., Longtin A., Galdrikian B., Farmer J.D. // Physica D. 1992. V. 58. N 1-4. P. 77-94.

[10] Корнилов М.В., Сысоев И.В. // Изв. вузов. Прикладная нелинейная динамика. 2014. Т. 22. № 4. С. 66-75.

[11] Schwarz G. // Annals Statist. 1978. V. 6. N 2. P. 461-464.

[12] Kornilov M.V., Medvedeva T.M., Bezruchko B.P., Sysoev I.V. // Chaos, Solit. Fract. 2016. V. 82. P. 11-21.

[13] Дмитриев А.С., Кислов В.Я. // Радиотехника и электроника. 1984. Т. 29. № 12. C. 2389-2398.

[14] Ikeda K., Matsumoto K. // Physica D. 1987. V. 29. N 1-2. P. 223-235.

Письма в ЖТФ, 2018, том 44, вып. 10 